\title{
Upgrade possibilities for continuous wave rf electron guns based on room-temperature very high frequency technology
}

\author{
F. Sannibale, ${ }^{1, *}$ D. Filippetto, ${ }^{1}$ M. Johnson, ${ }^{1}$ D. Li,${ }^{1}$ T. Luo, ${ }^{1}$ C. Mitchell, ${ }^{1}$ J. Staples, ${ }^{1}$ \\ S. Virostek, ${ }^{1}$ R. Wells, ${ }^{1}$ and J. M. Byrd ${ }^{2}$ \\ ${ }^{1}$ Lawrence Berkeley National Laboratory, One Cyclotron Road, Berkeley, California 94720, USA \\ ${ }^{2}$ Argonne National Laboratory, 9700 Cass Avenue, Lemont, Illinois 60439, USA
}

(Received 31 August 2017; published 27 November 2017)

\begin{abstract}
The past decade was characterized by an increasing scientific demand for extending towards higher repetition rates $(\mathrm{MHz}$ class and beyond) the performance of already operating lower repetition rate accelerator-based instruments such as x-ray free electron lasers (FELs) and ultrafast electron diffraction (UED) and microscopy (UEM) instruments. Such a need stimulated a worldwide spread of a vibrant R\&D activity targeting the development of high-brightness electron sources capable of operating at these challenging rates. Among the different technologies pursued, rf guns based on room-temperature structures resonating in the very high frequency (VHF) range (30-300 MHz) and operating in continuous wave successfully demonstrated in the past few years the targeted brightness and reliability. Nevertheless, recently proposed upgrades for x-ray FELs and the always brightness-frontier applications such as UED and UEM are now requiring a further step forward in terms of beam brightness in electron sources. In this paper, we present a few possible upgrade paths that would allow one to extend, in a relatively simple and cost-effective way, the performance of the present VHF technology to the required new goals.
\end{abstract}

DOI: 10.1103/PhysRevAccelBeams.20.113402

\section{INTRODUCTION}

Electron beam applications such as free electron lasers (FELs), light sources based on energy recovery linacs, inverse Compton scattering sources, and ultrafast electron diffraction (UED) and microscopy (UEM), all require electron sources capable of generating beams with high transverse brightness and a properly controlled longitudinal phase space. Although the actual optimization for such beam quantities depends on the particular application, in all cases the ultimate performance of the application itself is essentially set by the highest brightness and best control of the longitudinal phase space allowed by the electron source.

For the case of relatively low repetition rate $(100 \mathrm{~Hz}-$ class) applications, a number of radio frequency (rf) gun schemes, based on high frequency ( $\gtrsim 1 \mathrm{GHz}$ ) room temperature resonant structures (cavities) and on photocathodes, have already demonstrated the required performance level to operate an X-ray FEL (notably transverse normalized emittances ranging from about 0.2 to $0.6 \mu \mathrm{m}$ for charges per bunch ranging from about 10 to $300 \mathrm{pC}$-a more complete list of parameter requirements can be found elsewhere [1]). While successful, such a gun technology

\footnotetext{
fsannibale@lbl.gov
}

Published by the American Physical Society under the terms of the Creative Commons Attribution 4.0 International license. Further distribution of this work must maintain attribution to the author(s) and the published article's title, journal citation, and DOI. cannot be directly scaled up to allow for repetition rates higher than $\sim 1 \mathrm{kHz}$. Indeed, the extremely high heat load that higher repetition rates would impose on the cavity walls is well beyond any cooling system capability.

On the other hand, science demand in the past decade pushed for much higher repetition rates, and in particular, a large class of experiments was claiming dramatic benefits from x-ray FEL and UED/UEM facilities operating at MHz-class repetition rates [2]. In response to this, a number of groups around the world started to study and develop alternative technologies and schemes that could generate high brightness guns capable of operating at such challenging repetition rates [3]. The effort paid off and in the past few years, several of these schemes were able to demonstrate the performance required by the present generation of high repetition rate applications. For example, the beam performance required at $\sim 1 \mathrm{MHz}$ repetition rate by LCLS-II, the $\mathrm{x}$-ray FEL under construction at SLAC (the Stanford Linear Accelerator Center [4]), was experimentally demonstrated by a direct current $(\mathrm{dc})$ gun scheme at Cornell [5], and by the very high frequency gun, shortly referred to as the very high frequency (VHF) gun, a lower frequency $(\sim 186 \mathrm{MHz})$ room-temperature rf gun developed by our group at the Lawrence Berkeley National Laboratory (LBNL) [6]. As a consequence of these results, a close version of the present VHF gun has been selected as the electron source for the LCLS-II, and is in the final fabrication stage at LBNL.

Although the basic gun requirements for the operation of high repetition rate applications have been already demonstrated, FELs, and brightness-frontier applications 


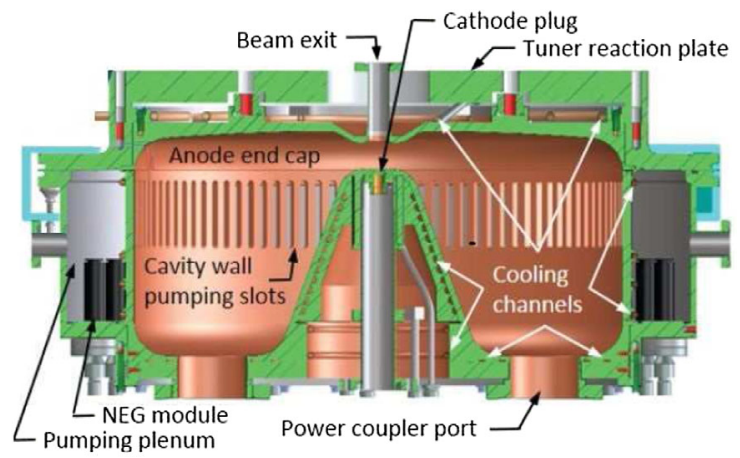

FIG. 1. A CAD cross section of the existing APEX VHF gun.

such as UED and UEM, would strongly benefit, as recently pointed out by a DOE-BES workshop on future electron sources [7], by the availability of sources with even higher transverse brightness (i.e. lower normalized emittances) and improved longitudinal phase space control capability. For example, $100 \mathrm{pC}$ bunches with transverse normalized emittances approaching $0.1 \mu \mathrm{m}$ would allow for an extension of the lasing spectra in FELs towards much shorter wavelengths (see for example Figs. 1-3 in [7]). Also, at the much lower charges per bunch used in UED/UEM experiments, a higher brightness would directly translate into higher spatial/time resolution and shorter acquisition times. This demand is evidence of the need for the development of brighter high repetition rate electron guns.
The brightness enhancement can be achieved with schemes that allow for higher accelerating fields at the cathode and higher beam energies at the gun exit.

Higher fields at the cathode during electron emission directly impact the maximum brightness obtainable by a gun. Indeed, for beams with transverse beam sizes much larger than the longitudinal (the so-called "pancake beams"), the maximum $4 \mathrm{D}$ transverse brightness $B_{\max }$ is proportional to [8]

$$
B_{\max } \propto \frac{E_{z}^{\text {cathode }}}{\Delta \mathcal{E}_{\text {cathode }}},
$$

and for the case of beams with the inverse size aspect ratio ("cigar beams") the maximum brightness is proportional to [9]

$$
B_{\max } \propto \frac{\left(E_{z}^{\text {cathode }}\right)^{3 / 2} \sigma_{\tau}}{\sqrt{\sigma_{r}} \Delta \mathcal{E}_{\text {cathode }}}
$$

where $E_{z}^{\text {cathode }}$ is the accelerating electric field at the cathode during electron emission, $\Delta \mathcal{E}_{\text {cathode }}$ is the "excessive energy," a quantity that depends on the type of cathode used and on the emission process, $\sigma_{\tau}$ is the rms bunch length in time units, and $\sigma_{r}$ is the rms beam size at the cathode. (a)

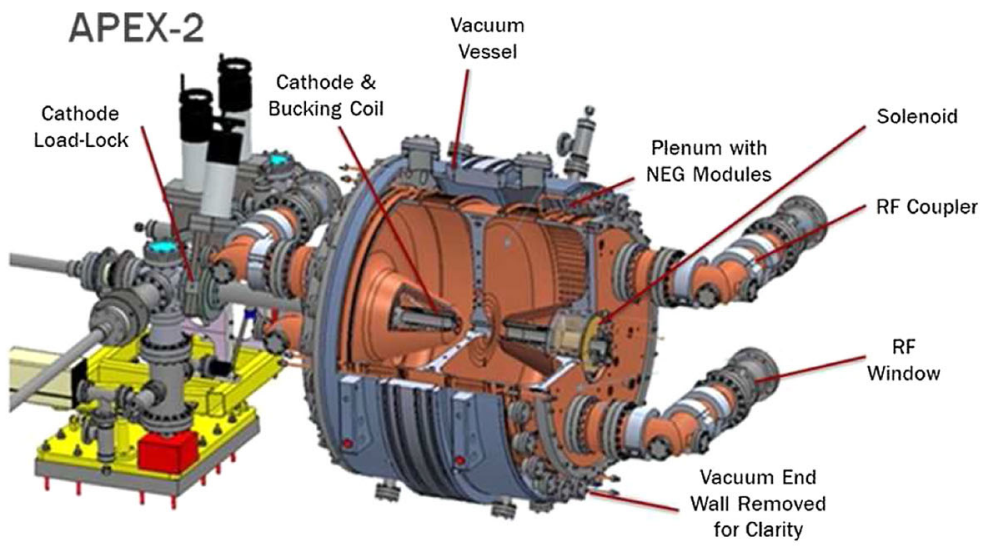

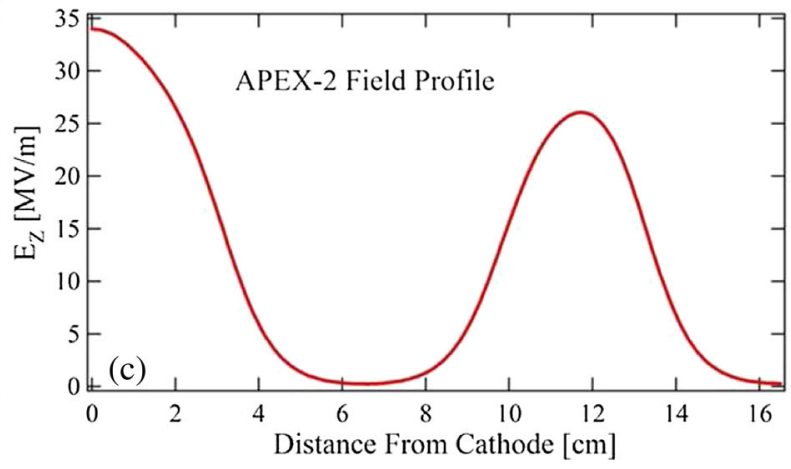

FIG. 2. (a) 3D CAD view of a double-cell version of APEX and of its main parts. (b) Electric field distribution inside the cells. (c) Longitudinal electric field intensity along the longitudinal axis of the gun. 

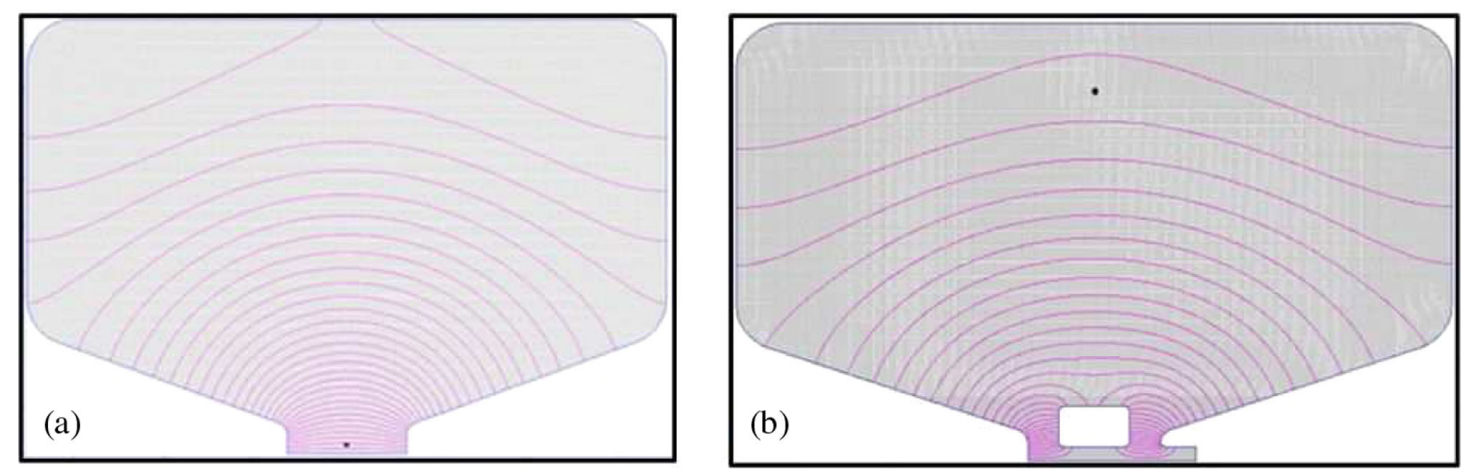

FIG. 3. Electric field distributions for two additional APEX-2 configuration options. (a) Single-cell with dual-reentrant nosecones. (b) Single cell with drift tube. The design field at the cathode for both configurations is $\sim 35 \mathrm{MV} / \mathrm{m}$. See the text for more details.

The above expressions show that in both regimes, higher fields at the cathode mean higher brightness.

Moreover, higher fields at the cathode in combination with higher beam energies at the gun exit, allow for "quickly" accelerating the beam to relativistic energy, minimizing emittance dilution and longitudinal phasespace distortions due to space charge forces. Such distortions, if not properly controlled, can severely affect the bunch compression capability required by FEL schemes for achieving the kA-class peak currents necessary for an efficient lasing process.

In summary, for a fixed charge per bunch, higher fields at the cathode during emission and higher beam energies at the gun exit result in beams with a smaller transverse emittance (i.e. with a higher transverse brightness) and with a more linear longitudinal phase space.

In this paper, we present and discuss possible upgrade options that would allow one to extend the VHF-gun performance towards these new goals.

\section{CHARACTERISTICS OF THE VHF-GUN SCHEME}

In a gun scheme based on VHF technology, the core of the system is represented by a room temperature normal conducting (NC) copper cavity resonating in the very high frequency (VHF) range (30-300 MHz). With respect to $\gtrsim 1 \mathrm{GHz}$ low repetition rate rf guns, the lower frequency allows for a favorable scaling, where the power density in the walls is dramatically decreased while the reduction of the accelerating field at the cathode is contained at an acceptable level. The reduced wall power allows for the cavity to operate in continuous wave $(\mathrm{cw})$ mode with field intensities still sufficient for the generation of highbrightness electron beams [10]. The VHF-gun scheme offers a number of additional appealing features: it relies on mature rf and mechanical technologies ensuring both reliability and a reduced system complexity when compared with the dc and superconducting rf (SRF) cases; it can operate at energies and accelerating fields at the cathode, much higher than the ones in dc guns and higher than in the presently operating SRF guns; and it is capable of the extremely low vacuum pressures needed to operate the high quantum efficiency $(\mathrm{QE})$ semiconductor cathodes necessary for achieving the average currents required by high repetition rate FELs.

Indeed, all such characteristics have been already successfully demonstrated by the first VHF gun developed at LBNL in the framework of the APEX project $[11,12]$. A CAD cross section of such a gun, which operates at $186 \mathrm{MHz}$ (seventh subharmonic of $1.3 \mathrm{GHz}$, the frequency of the dominant SRF linac technology), is shown in Fig. 1.

TABLE I. Existing APEX VHF-gun parameters: Design and demonstrated values.

\begin{tabular}{lccc}
\hline \hline Parameter & Design value & Measured value & Units \\
\hline Operation mode & $\mathrm{cw}$ & $\mathrm{cw}$ & \\
Frequency & 185.7 & 185.7 & $\mathrm{MHz}$ \\
Nominal gap voltage & 0.75 & up to $\sim 0.8$ & $\mathrm{MV}$ \\
$Q_{0}$ & 31600 (ideal conductor) & 30040 & \\
Max electric field at cathode & 19.5 & $\mathrm{Up}$ to $\sim 22$ & $\mathrm{MV} / \mathrm{m}$ \\
Peak surface field at $0.75 \mathrm{MV}$ & 24.1 & Not measured & $\mathrm{MV} / \mathrm{m}$ \\
rf power for 0.75 MV & $<100$ & $\sim 90$ & $\mathrm{~kW}$ \\
Peak wall power density at $0.75 \mathrm{MV}$ & 25 & Not measured & $\mathrm{W} / \mathrm{cm}^{2}$ \\
Operation pressure at $0.75 \mathrm{MV}$ & $10^{-10}-10^{-9}$ & $\sim 5 \times 10^{-10}$ & $\mathrm{Torr}$ \\
Dark current at $0.75 \mathrm{MV}$ & $<1$ & $<10^{-3}$ & $\mu \mathrm{A}$ \\
\hline \hline
\end{tabular}


Table I shows the gun main parameters while a detailed description of the gun fabrication can be found in [13]. After the completion of the beam tests that demonstrated beams with the characteristics required to operate a high repetition rate FEL [6], the APEX VHF gun is now used at LBNL as the source for high repetition rate UED experiments in the framework of the HiRES project [14].

The new VHF gun being built at LBNL for the LCLS-II injector presents some modified features with respect to the APEX VHF gun. The cooling scheme for the anode end cup of the gun cavity (see Fig. 1) was modified to improve the heat transfer between the cavity wall and the cooling channels. This allows for a decrease of the anode end cup temperature during operation reducing outgassing from the wall with a beneficial effect on the vacuum pressure. The tuner system was also modified to extend (double) the range of tunability of the cavity frequency. Other minor modifications were implemented and are described in more detail in [13].

A VHF gun, with characteristics similar to the APEX one, was built and tested at low rf power at SINAP in Shanghai [15], and the baseline injector of the recently approved Shanghai Coherent Light Facility (SCLF), a cw $\mathrm{x}$-ray FEL in China, includes a VHF gun as the electron source [16].

It is important to remark that during the extensive tests at LBNL, the APEX VHF gun did not show any sign of voltage breakdown associated with the high electric fields present in the gun cavity. Values of up to $\sim 22 \mathrm{MV} / \mathrm{m}$ at the cathode were routinely used without issues, and the initial rf conditioning of the gun to full power was extremely fast ( $\sim 2$ days) with no arcing events and with the progress rate essentially limited by vacuum conditioning.

Additionally, extremely low levels of field emission were observed in the gun during its high-field operation with $\sim 0.1 \mathrm{nA}$ "dark current" measured at the nominal operational power. This figure compares well, for example, with the LCLS-II requirement for a dark current smaller than $400 \mathrm{nA}$.

These two observations are a clear indication that there is still a significant margin for increasing the field in the cavity, and this consideration is at the base of the proposed upgrade paths described in the next sections.

\section{POSSIBLE VHF-GUN CW TECHNOLOGY UPGRADE PATHS}

In the previous section it was pointed out that the higher brightness upgrade of the gun must provide higher fields at the photocathode and higher output energies in order to obtain the desired beam quality improvement. In this paper, we will refer to the upgraded gun options as APEX-2.

The present APEX VHF-gun cavity design was highly optimized for maximum shunt impedance consistent with no multipacting tendencies at the nominal operating gradient. With the success and experience gained with the APEX gun, an obvious extension would be to place two APEX-like guns back to back, with the nosecones facing a diaphragm between them, with the two cavities uncoupled from each other and separately driven. This scheme would keep the same field at the cathode while doubling the output energy of the APEX VHF- gun to $\sim 1.5 \mathrm{MeV}$.

In order also to increase the field on the photocathode it is necessary to operate at a higher peak wall power density than $25 \mathrm{~W} / \mathrm{cm}^{2}$. We will pursue that direction, but in order to maintain the wall peak power density at levels that can be safely handled by conventional cooling schemes, we will also slightly decrease the resonant frequency from 185.7 MHz of APEX to $162.5 \mathrm{MHz}$ (respectively the seventh and eighth subharmonic of $1.3 \mathrm{GHz}$, the dominant SRF technology frequency). The lower frequency increases the skin depth of the circulating rf current on the wall, and for a constant field integral along the axis, the wall power density goes as the frequency to the $5 / 2$ power [17]. In addition, the $162.5 \mathrm{MHz}$ choice allows to benefit by commercially available rf power sources at this frequency and it is compatible with existing superconducting linac cavities at $325 \mathrm{MHz}, 650 \mathrm{MHz}$ and $1.3 \mathrm{GHz}$.

Table II shows the goal parameters targeted by the APEX-2 design compared to the present APEX gun. The same requirements, shown in Table I in terms of vacuum and dark current, also apply to the APEX-2 case.

Radio frequency calculations indicated that using the double-cell concept and lowering the frequency to 162.5 $\mathrm{MHz}$ allows for the field at the photocathode to be raised to about $34 \mathrm{MV} / \mathrm{m}$ and for a beam energy of $\sim 2 \mathrm{MV}$ while maintaining a modest wall power density of

TABLE II. Present APEX VHF-gun actual parameters vs APEX-2 goal parameters.

\begin{tabular}{lccc}
\hline \hline Parameter & APEX & APEX-2 & Units \\
\hline Operation mode & $\mathrm{cw}$ & $\mathrm{cw}$ & \\
Frequency & $186.7(1300 / 7)$ & $162.5(1300 / 8)$ & $\mathrm{MHz}$ \\
Technology & Room-temperature $\mathrm{Cu}$ & Room-temperature $\mathrm{Cu}$ & \\
Number of cells & 1 & 1 or 2 & $\mathrm{~W} / \mathrm{cm}^{2}$ \\
Peak power density & 22 & $<35$ & $\mathrm{~kW}$ \\
Maximum rf power & 120 & $<250$ & $\mathrm{MV} / \mathrm{m}$ \\
Launching field at photocathode & $\sim 20$ & $\sim 35$ & {$[\mathrm{MeV}]$} \\
Beam energy & 0.75 & $1.5-2.0$ & \\
\hline \hline
\end{tabular}


$30 \mathrm{~W} / \mathrm{cm}^{2}$. About $250 \mathrm{~kW}$ of rf power are required to operate the gun in this condition. As a consequence of the lower frequency, the gun cavity radius increases from the $36 \mathrm{~cm}$ of the APEX VHF gun to the $47.5 \mathrm{~cm}$ of this new double-cell design. The gap length in each cell is maintained at $4 \mathrm{~cm}$. A 3D CAD view of this double-cell case is shown in Fig. 2 with the field distribution in the cells and the field profile along the longitudinal axis of the gun calculated with the SUPERFISH code [18]. It is worthwhile to remark that the designs for this configuration and for those that will be described later in this section are preliminary, and that the final cavity size and shape will be optimized for maximizing the cavity shunt impedance, minimizing multipacting resonances, and easing the mechanical fabrication.

The figure also shows the vacuum load-lock system used for exchanging cathodes without breaking vacuum and the four high power rf couplers (two per cell) used to feed the power into the cells. All these components are exact copies of the well-tested and reliable parts used in APEX. The same vacuum scheme, successfully used in APEX, will be also adopted for the APEX-2 case. A large number of slots on the walls of each of the cells (visible in the figure) creates, with negligible field distortion, a high vacuum conductance path for residual gas molecules towards a plenum where a number ( 20 per cell) of nonevaporablegetter modules are located providing an exceptional pumping capability. In a potentially different way than in APEX, where a mechanical system for the cavity frequency tuneup is used, in APEX-2 the tuning of the two cells will be probably performed by differentially changing the temperature of the cavity parts by a dynamic variation of their cooling water flow. Additional studies will confirm the use of this or of an alternative tuning scheme. It is also worthwhile to point out that with the two uncoupled cells, the phasing and rf amplitude tuning of each cell are independent, allowing for some potentially interesting beam phase space manipulation (beam loading compensation, energy chirping for compression, etc.). The bucking and focusing solenoids can be located close to the cathode (inside the first and second nosecones respectively) with beneficial impact on beam dynamics.

A variation of this back-to-back scheme consists in eliminating the diaphragm between the two cells (making effectively a larger single cell cavity with two reentrant nosecones and an $8 \mathrm{~cm}$ gap). In this configuration, whose field distribution is shown in the part (a) of Fig. 3, the power lost on the central diaphragm is eliminated, so the total $\mathrm{rf}$ drive power required to obtain the same fields in cavity is reduced, or, if kept constant with a slightly higher wall power density, a higher output energy is obtained. The down side of this configuration is that the field at the photocathode is reduced and the advantage of having independent phasing between two cells is lost. In summary, this single-cell configuration can produce a higher output energy at the expense of a relatively lower field on the photocathode, compared to the back-to-back double-cell configuration previously described.

A third hybrid variation takes the best features of both configurations discussed above to maintain the highest field on the photocathode and maximize the output energy. In this version, the diaphragm between the two cells of the back-to-back double-cell configuration is still eliminated but a drift tube, suspended on four stems, is inserted between the nosecones. The field on the photocathode in this configuration, visible in part (b) of Fig. 3, is now increased to $36 \mathrm{MV} / \mathrm{m}$ when $\sim 200 \mathrm{~kW}$ rf power is applied to the cavity. In this example, the entrance of a $7 \mathrm{~cm}$ drift tube, with a $1.5 \mathrm{~cm}$ aperture radius, is placed $3 \mathrm{~cm}$ from the cathode. In this geometry, the center of the second gap, which is also $3 \mathrm{~cm}$ long, is located at $10 \mathrm{~cm}$ from the center of the first gap, or about $21 \mathrm{rf}$ degrees for a bunch crossing through the drift tube with about a $1 \mathrm{MeV}$ energy. This will induce a small phase slip that reduces the beam energy by about $4 \%$, which would still be approximately $2 \mathrm{MeV}$ at the gun exit if the beam is generated -10.5 degrees off crest at the cathode. For a fixed rf power, this configuration gives the highest energy and the highest field on the photocathode. The drift tube would be supported on four thin stems to eliminate dipole or quadrupole distortions of the $\mathrm{rf}$ field. As this is an overdetermined mechanical configuration, problems with heating and expansion of the stems can be remedied by attaching the stems with a tangential component to the drift tube, which will then rotate with stem expansion, but not be displaced longitudinally. Additionally, if beam dynamics studies indicate that it is necessary, the drift tube could include an imbedded focusing electromagnetic solenoid (with current feeding wires twisted together inside one of the stems to cancel undesired magnetic fields).

Most of the mechanical, vacuum and rf solutions used for the double-cell option described at the beginning of this section can also be applied to both the single-cell versions with and without drift tube.

We are also planning to explore the option of cryogenic operation for APEX-2. At cryogenic temperature, both the electric conductivity and thermal conductivity of the cavity copper walls increase significantly, which alleviates the thermal loading and improves the cooling efficiency. Additionally, recent experimental results showed an increase of the copper electric rigidity at low temperature significantly reducing the orf breakdown rate [19].

\section{BEAM DYNAMICS PERFORMANCE}

In order to evaluate the beam dynamics performance of the APEX-2 VHF guns, we carried out initial simulations with the ASTRA code [20] using an APEX-like injector layout, visible in Fig. 4. The original APEX gun was alternatively replaced by two of the different versions of the previously described APEX-2 guns: the double-cell and the single-cell dual-reentrant nosecones. Layouts with 


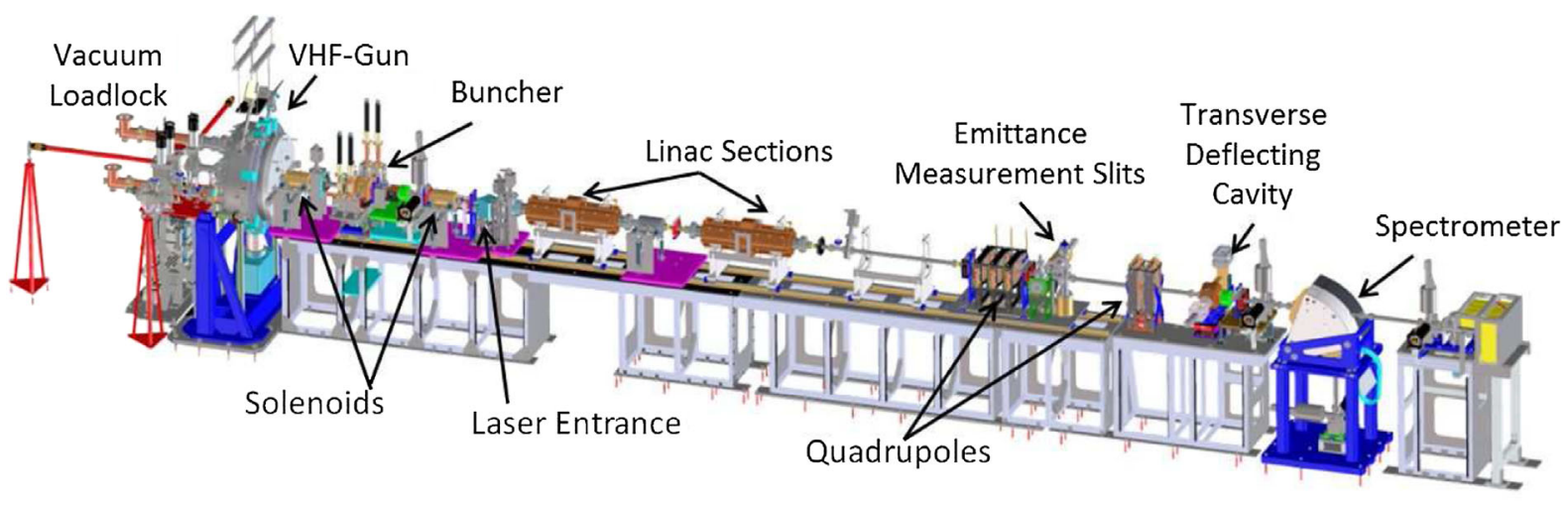

FIG. 4. APEX-like injector layout used for the beam dynamics simulations.

and without a buncher cavity between the two solenoids were also investigated during the simulations.

Figure 5 shows the results of a multiobjective genetic algorithm (MOGA) optimization for the case of $100 \mathrm{pC}$ charge per bunch (a value of interest for FEL applications) used to simultaneously minimize the normalized emittance

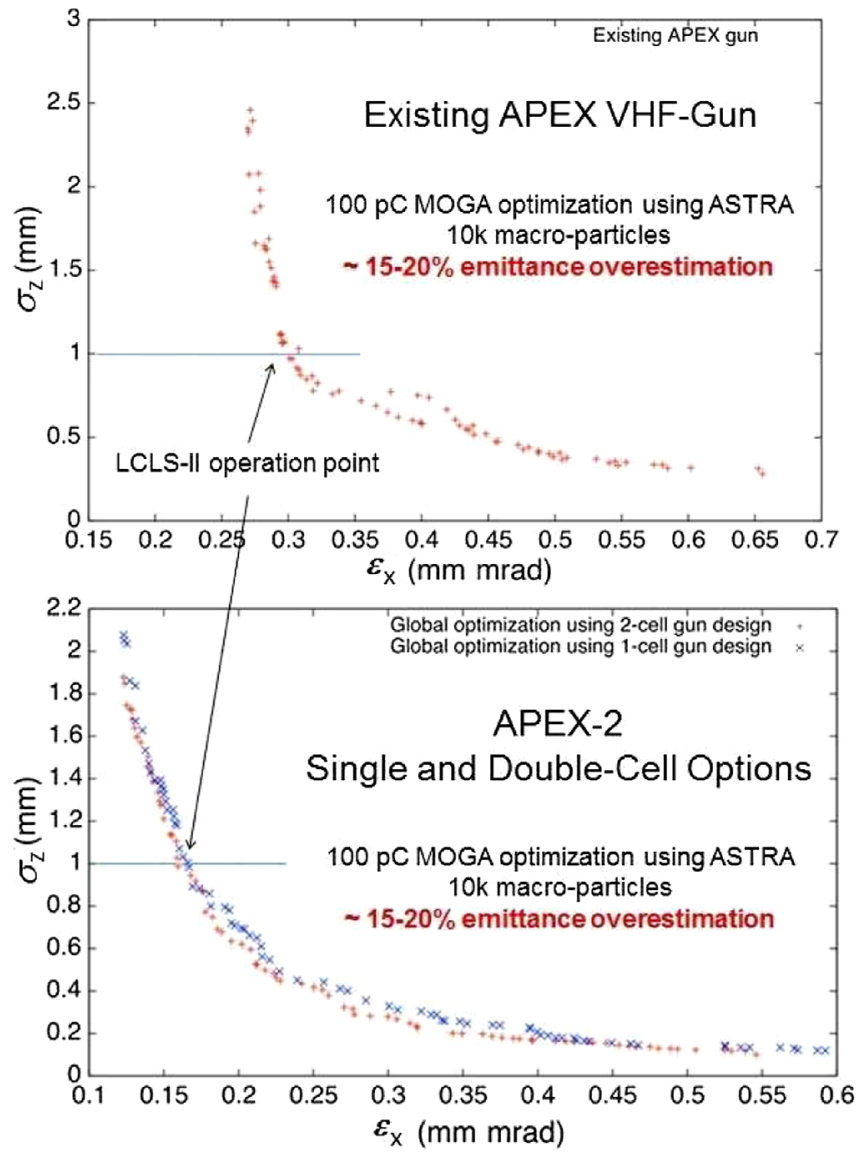

FIG. 5. Fronts of $100 \mathrm{pC}$ solutions illustrating the trade-off between emittance (100\% of particles included) and rms bunch length at the end of the injector. Top: Present APEX VHF-gun case. Bottom: The fronts for the cases of the double cell and single cell with no drift tube APEX-2 configurations described in the text. and bunch length (and hence the peak current) at the end of the injector. Results are shown for the present APEX VHF gun at the top, and for the two APEX-2 gun configurations at the bottom. For the APEX-2 cases, the field at the cathode was kept fixed at $34 \mathrm{MV} / \mathrm{m}$, no buncher was used, and the energy at the injector exit was approximately $15 \mathrm{MeV}$. The Pareto-optimal fronts in the figure show solutions illustrating the trade-off between low emittances and short bunch lengths. The extremely similar Paretooptimal fronts for the two APEX-2 cases indicate that for similar cathode fields the performance of the gun is largely independent of the gun geometry. All the simulations shown in the figure used $10 \mathrm{k}$ macroparticles in order to keep computing time reasonable. Accurate simulations using 250k macroparticles, performed on a few characteristic solutions taken from the fronts in Fig. 5, systematically showed emittance values about $15 \%-20 \%$ smaller than those shown in the figure. In the bunch length range of interest for an FEL like LCLS-II (between 0.8 and $1.2 \mathrm{~mm}$ $\mathrm{rms}$ ), the simulated normalized emittance values for $100 \%$ of the particles are between 0.12 to $0.144 \mu \mathrm{m}$. Figure 6 shows details of a particular (250k macroparticle) solution selected from the Pareto-optimal front for the APEX-2 double-cell case shown in Fig. 5. This preliminary solution already satisfies the enhanced requirements at the exit of a cw FEL injector operating at $100 \mathrm{pC}$ charge per bunch. The plots in the top left and bottom left of the figure show a peak current of $\sim 13 \mathrm{~A}$ and the relatively flat longitudinal phase space in the core of the beam necessary to achieve the required compression and peak currents at the FEL undulator entrance. Note that the linear and quadratic correlations in the longitudinal phase space have been removed in this figure, because they can be compensated in the FEL main linac (for example, by dephasing the rf in some of the linac cavities and by using harmonic cavities). The largely constant and well matched slice emittance along the beam (respectively visible in the top right and bottom right of Fig. 6) indicates a successful emittance compensation process that allows for a final projected normalized emittance of $0.119 \mu \mathrm{m}$ for $95 \%$ of the particles. The asymmetric longitudinal profile of the beam is the result 


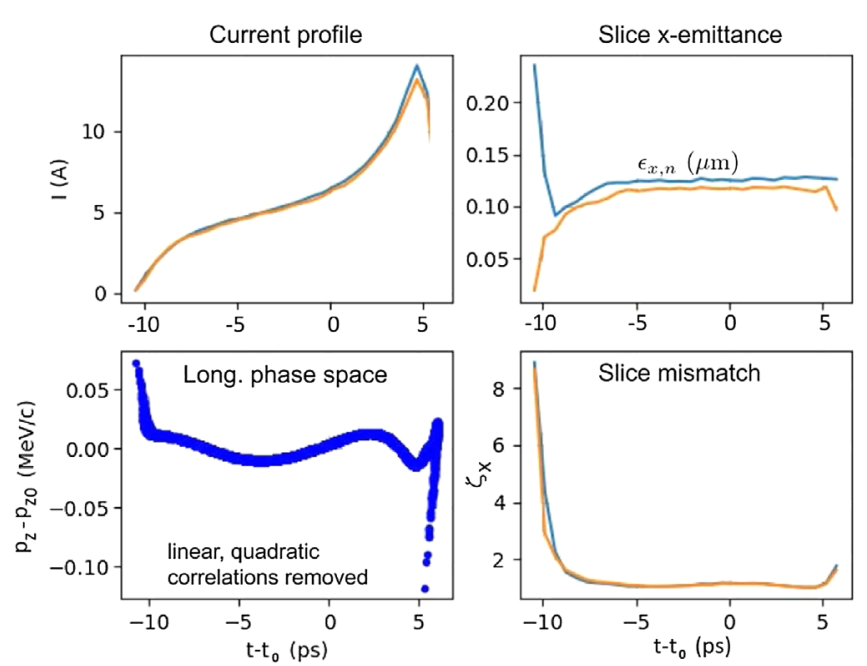

FIG. 6. High resolution simulation using ASTRA with $250 \mathrm{k}$ macroparticles showing details of a particular $100 \mathrm{pC}$ solution selected from the optimization front shown in Fig. 5 for the double-cell APEX-2 configuration case. The blue and orange curves indicate the results for $100 \%$ and $95 \%$ of the particles respectively.

of the compression performed by dephasing the two accelerating sections in the APEX layout. More symmetric solutions can be obtained by using a layout that includes a buncher cavity upstream of the accelerating sections as visible in Fig 4.

These initial results show a decrease in emittance per plane approaching a factor 2 with respect to the values obtainable with the present APEX VHF gun, resulting in a remarkable approximately fourfold increase of brightness.

\section{CONCLUSIONS}

Demand from existing and proposed high-repetition rate facilities for higher brightness electron beams is requiring the design/upgrade of guns with higher accelerating fields and beam energies.

The studies presented in this paper indicate the possibility of upgrading the successful room-temperature continuous wave VHF-gun technology to higher accelerating fields at the cathode and electron beam energies while maintaining the reliability and vacuum performance already demonstrated by the original VHF gun of the APEX project at the Lawrence Berkeley National Laboratory. Several possible options were analyzed and studied at the conceptual level, indicating no evident show stoppers. The results indicated that fields as high as $\sim 35 \mathrm{MV} / \mathrm{m}$ at the cathode and energies up to $\sim 2 \mathrm{MeV}$ at the gun exit can be obtained with less than $\sim 250 \mathrm{~kW}$ rf power. Such field and energy values are four-five times higher than the ones obtainable by dc guns, and comparable to those presently targeted by the $R \& D$ activities on superconducting rf guns but without the costs and complexity associated with those types of schemes.
Initial simulations indicate an approximately fourfold brightness increase with respect to that obtainable with the present APEX VHF- gun. Such a performance leap would allow for photon spectra extended to much shorter wavelengths in X-ray FEL applications and to higher spatial/time resolutions and shorter acquisition times in ultrafast electron diffraction and microscopy experiments.

\section{ACKNOWLEDGMENTS}

The authors would like to thank Qiang Gu for useful discussions. This work was supported by the Director of the Office of Science of the U.S. Department of Energy under Contract No. DEAC02-05CH11231.

[1] F. Sannibale, High-brightness high-duty cycle electron injectors, Nucl. Instrum. Methods Phys. Res., Sect. A 740, 10 (2014).

[2] See, for example, E. Arenholz et al., in Toward Control of Matter: Basic Energy Science Needs for a New Class of X-Ray Light Sources, Berkeley, CA, 2008; also LBNL Report No. LBNL-1034E, 2008.

[3] See, for example, references in F. Sannibale, Overview of Electron Source Development for High Repetition Rate FEL Facilities, in Proceedings of the 2016 North America Particle Accelerator Conference, Chicago IL, USA (2016), p. 445.

[4] J. Galayda, The Linac Coherent Light Source-II Project, in Proceedings of the 2014 International Particle Accelerator Conference, Dresden, Germany (2014), p. 935.

[5] A. Bartnik, C. Gulliford, I. Bazarov, L. Cultera, and B. Dunham, Operational experience with nanocoulomb bunch charges in the Cornell photoinjector, Phys. Rev. ST Accel. Beams 18, 083401 (2015).

[6] F. Sannibale et al., APEX Phase-II Commissioning Results at the Lawrence Berkeley National Laboratory, in Proceedings of the 2016 International Particle Accelerator Conference, Busan, South Korea (2016), p. 1041.

[7] Available at https://science.energy.gov/ /media/bes/pdf/ reports/2017/Future_Electron_Source_Worskhop_Report .pdf.

[8] I. V. Bazarov, B. M. Dunham, and C. K. Sinclair, Phys. Rev. Lett. 102, 104801 (2009).

[9] D. Filippetto, P. Musumeci, M. Zolotorev, and G. Stupakov, Maximum current density and beam brightness achievable by laser-driven electron sources, Phys. Rev. ST Accel. Beams 17, 024201 (2014).

[10] J. Staples, F. Sannibale, and S. Virostek, CBP Technical Note No. 366, 2006, LBNL-1003792 (available at https:// publications.lbl.gov/publication_search).

[11] F. Sannibale et al., Advanced photoinjector experiment photogun commissioning results, Phys. Rev. ST Accel. Beams 15, 103501 (2012).

[12] D. Filippetto, H. Qian, and F. Sannibale, Cesium telluride cathodes for the next generation of high-average current high-brightness photoinjectors, Appl. Phys. Lett. 107, 042104 (2015). 
[13] R. P. Wells, W. Ghiorso, J. Staples, T. M. Huang, F. Sannibale, and T.D. Kramasz, Mechanical design and fabrication of the VHF-gun, the Berkeley normalconducting continuous-wave high-brightness electron sourc, Rev. Sci. Instrum. 87, 023302 (2016).

[14] D. Filippetto and H. Qian, Design of a high-flux instrument for ultrafast electron diffraction and microscopy, J. Phys. B 49, 104003 (2016).

[15] Q. Gu, L.-P. Chen, G.-Q. Lin, and W.-C. Fang, VHF Gun Research at SINAP, in Proceedings of IPAC2013 (JACoW, Shanghai, China, 2013), p. 380.
[16] Shanghai Coherent Light Facility (SCLF) Pre-Conceptual Design Report, 2017.

[17] J. Staples, CBP Technical Note No. 395, 2007, LBNL-1003793 (available at https://publications.lbl.gov/ publication_search).

[18] http://laacg.lanl.gov/laacg/services/download_sf.phtml.

[19] J. B. Rosenzweig et al., Next generation high brightness electron beams from ultrahigh field cryogenic radio frequency photocathode sources, arXiv:1603.01657.

[20] K. Flottman, http://www.desy.de/ mpyflo/astradokumen tation/. 